\title{
AACE Member Engagement Lounges During COVID-19
}

\author{
James Marmion $^{1}$
}

Accepted: 1 April 2021 / Published online: 13 April 2021

(C) American Association for Cancer Education 2021

Over the past year, we have had to learn new styles of working and fresh ways of reaching out to others. As cancer educators, we have faced some very specific challenges in many aspects of our work: how to connect with our patients and administer virtual physical exams; how to teach our students; how to redefine our objectives; and how we might simply share our ideas with one another. In short, we have had to re-think the way we do business. One positive element we can take from this year of isolation is that it has given us more time to think. We have moved on from those early, reactive cancelations of our activities and into an era where we have accepted - even embraced - the need for change and proactively shaped new working structures that better serve our needs. This has reaped some benefits, especially in the way that our collective isolation has been the catalyst for our embrace of the virtual working environment. As and when (please, when!) we see the COVID threat recede, it is a given that we will still be thinking carefully about virtual ways to carry out our work: whether to travel is less efficient than to teleconference, ways we can reinforce student online learning, or how we can enhance our virtual outreach to the public.

One of the AACE's new methods of working has been the development of Zoom-based member Engagement Lounges. They were explicitly initiated as a reaction to the COVID-19 pandemic, focusing on the work of our members and as a way to help them face the challenges the pandemic presented to us. AACE's Membership Committee set up the first lounge in April 2020, just a month after most of our lives became locked down. One year later, we are about to host our sixth, and there is every prospect that we will grow and maintain them as a regular feature going forward.

The great strength of the Engagement Lounge format has been its adaptability. After all, it is simply a virtual gathering

James Marmion

james.marmion@stjude.org

1 St. Jude Children's Research Hospital, 262 Danny Thomas Place, Memphis, TN 38105, USA of people, with some of them leading and directing a conversation. We have used this flexibility to host lounges in a series of different formats, each suited to our audience, the subject at hand, and the outcomes we want to achieve.

The first Engagement Lounge 16 April 2020: NCIFunded Summer Programs During COVID-19 (recordings and moderator affiliations for the lounges are available at www.aaceonline.com/lounges) was in response to a practical and very pressing problem: with a country going into lockdown, was it going to be possible to host students in person over the summer? If not, could program directors offer any form of virtual alternative? At its core, this was a meeting of practitioners searching for common solutions. Later in the year, we hosted a second lounge on a similar theme 11 November 2020: NCI-funded Summer Programs: Tools and Best Practice for Virtual Programming. With COVID-19 established in our lives, the lounge looked ahead to the practicalities of running virtual summer programs in 2021. It also included representation from the $\mathrm{NCI}$ as a part of its own process of analyzing the changes it would need to introduce to keep such programs supported.

In May we hosted a lounge with a slightly different direction 14 May 2020: Using Cancer Education to Address Social Determinants of Health in the Era of COVID-19, as an opportunity to anticipate and highlight the primary theme of our 2020 conference. A collaboration with Emory University's Urban Health Initiative, the lounge featured moderated discussion on the topic at hand followed by an extended presentation by a guest speaker on the theme.

Our two subsequent lounges explored two sides of a similar coin: what practical measures educator-practitioners could take to adapt differing forms of education and outreach to a COVID-disrupted world. At the end of summer, 6 August 2020: Tools and Activities for Interactive/Applied Learning at a Distance, three practitioners presented their different experiences in hosting virtual education experiences for students, using presentations to highlight experiences followed by a moderated discussion. 
Then, early this year 10 February 2021: Cancer and COVID-19: Community Outreach and Engagement, a moderated panel discussion included two cancer center directors, a community outreach director, and the coordinator of a regional cancer resource center to discuss practical measures to maintain community outreach within the current climate.

Having run for a year, the Engagement Lounges have become an established AACE feature. This means we can now set up a schedule of lounges that draws from a broader canvas than the effect of the pandemic alone. This coming spring AACE's Education Committee will host a lounge focusing on how we measure program outcomes. That session will lay the groundwork for longer-term collaboration on the development of mechanisms to do this. Other topics we are planning include lessons learned from outreach to culturally specific communities and a session on ways we can engage health professionals-in-training in cancer education activities.

The Engagement Lounges are a service to our members but are also open to anybody who wishes to attend. Each lounge has been advertised not only to AACE members but also to those of partner organizations such as the Cancer Patient Education Network and the European Association for Cancer Education. Where we have had presenters or moderators from other organizations involved, they have been encouraged to share registration details as widely as they choose. In this way, we have sought to make the lounges as inclusive a tool as possible for raising the profile of AACE. The lounge on Community Outreach and Engagement was live-streamed on Facebook, with a positive secondary outcome of an increase in those following AACE through that network. We have recorded each of the lounges and made them available to our members through the AACE website. Attendance figures on the day have ranged from a low of 42 to a high of 140 , with the three others at 73, 87, and 97. The Facebookstreamed session remains accessible to all on AACE's Facebook page (facebook.com/aace1947).

In structure, the Engagement Lounges have ranged from one to two hours in length. Attendees enjoy several ways to interact with moderators and have their questions and opinions heard. In some segments live questions may be invited from the floor. However, most contributions come through the chat function, which allows questions and comments in a parallel written stream to the verbal presentations, with the secondary meeting moderator highlighting questions to be addressed by the primary moderator. With the April 2021 lounge, we will also begin using live AI-generated closed captioning during the broadcast to improve accessibility and to allow us to save a transcript of the lounge if desired.

A few days after each Engagement Lounge, AACE sends out a post-event survey to gain feedback from attendees. The overall average ratings (on a 5-point scale) have ranged from 3.69 to 4.63 . We have also received many detailed individual comments, which have not only been useful to the meeting hosts but also have allowed us to gauge which elements have been better received than others and make adjustments to later sessions accordingly.

One challenge we have faced has been how to translate the lounges into an ongoing or broader discussion. Professional colleagues can become enthused about a topic and be keen to interact further. This is an age-old issue for many professional organizations. We face a similar challenge with translating International Cancer Education Conference roundtable discussions into year-round engagement. Previous solutions have been shared email lists, open-access Google Documents, or invitations to share details on the post-event surveys. However, the organization has recently established AACE Connect, a community engagement site integrated with our database and website that allows discussion and file-sharing organized around specific themes. This should allow AACE to better organize ongoing contact and discussion around distinct subject areas.

In summary, having begun as a measure to support our members in adversity, the Engagement Lounges have become an established method of building community among AACE members and a tool to showcase AACE to the broader cancer education community.

James Marmion

St. Jude Children's Research Hospital, Office of Academic Programs in Biomedical Science

Member-at-Large, AACE Executive Council

Co-Chair, AACE Membership Committee

If there is a topic you would like to see discussed in a future Engagement Lounge, please contact james.marmion@stjude.org.

Publisher's Note Springer Nature remains neutral with regard to jurisdictional claims in published maps and institutional affiliations. 